\title{
Rheumatoid arthritis with pachymeningitis - a case presentation and review of the literature
}

\author{
Sanyukta Joshi ${ }^{1}$, Anna Masiak ${ }^{2}$, Zbigniew Zdrojewski ${ }^{2}$ \\ ${ }^{1}$ Student Clinical Rheumatology Circle, Medical University of Gdansk, Poland \\ 2Department of Internal Medicine, Connective Tissue Diseases and Geriatrics, Medical University of Gdansk, Poland
}

\begin{abstract}
Rheumatoid meningitis (RM) is a rare central nervous system (CNS) manifestation of rheumatoid arthritis (RA) with a wide spectrum of symptoms. We present a review of the literature with a rare illustrative case of a 61-year-old man with a history of seropositive rheumatoid arthritis (RA) who presented headaches, stroke-like symptoms and seizures. MRI revealed the leptomeningeal enhancement in the right hemisphere. As cerebromeningeal fluid showed increased level of protein and was positive for Candida mannan, the initial clinical diagnosis was fungal meningitis. Despite the antifungal treatment the patient's clinical condition did not improve. Detailed laboratory, radiologic and histopathological diagnostics enabled the diagnosis of RM.

In conclusion is worth to highlight that presentation of RM is variable and complex, diagnosing it is a big dilemma which is why it must be considered in the differential in a patient with long-standing seropositive RA.
\end{abstract}

Key words: rheumatoid arthritis, rheumatoid meningitis, stroke-like symptoms, central system involvement.

\section{Introduction}

Rheumatoid arthritis (RA) is a multifactorial, autoimmune systemic inflammatory disease which most commonly affects synovial joints. It presents with pain and stiffness of multiple joints which commonly involve proximal interphalangeal (PIP) and metacarpophalangeal (MCP) joints. The disease is estimated to affect $1 \%$ of the population predominantly women [1].

Rheumatoid arthritis has several extra-articular manifestations like rheumatoid vasculitis, nodules, interstitial pulmonary fibrosis, ocular manifestations (e.g. scleritis) or renal involvement. Nervous system manifestations of RA include peripheral neuropathies, mononeuritis multiplex, cervical myelopathy due to atlanto-axial subluxation and rheumatoid meningitis (RM) [2].

Rheumatoid meningitis is a rare central nervous system (CNS) manifestation of RA with symptoms ranging from headaches and seizures to deafness, speech impairment and stroke-like symptoms for instance hemi-paresis, cognitive impairment, focal neurological deficits which makes diagnosing it a challenge.

\section{Material and methods}

We analyzed studies reporting neurological manifestations of rheumatoid arthritis from PubMed and Google Scholar databases as a key words using a combination of search terms such as: rheumatoid arthritis with pachymeningitis.

Using a combination of presented search terms, we undertook a systematic review of the literature for discussion and analysis of studies reporting neurological manifestations of rheumatoid arthritis from PubMed and Google Scholar databases especially focusing on pachymeningitis.

\section{Case description}

A 61-year old man with speech impairment, left-sided paresis and generalized seizures was admitted to the

Address for correspondence:

Anna Masiak, Department of Internal Medicine, Connective Tissue Diseases and Geriatrics, Medical University of Gdansk, 7 Dębinki St., 80-211 Gdansk, Poland, e-mail: anna.masiak@wp.pl

Submitted: 14.01.2020; Accepted: 9.04.2020 

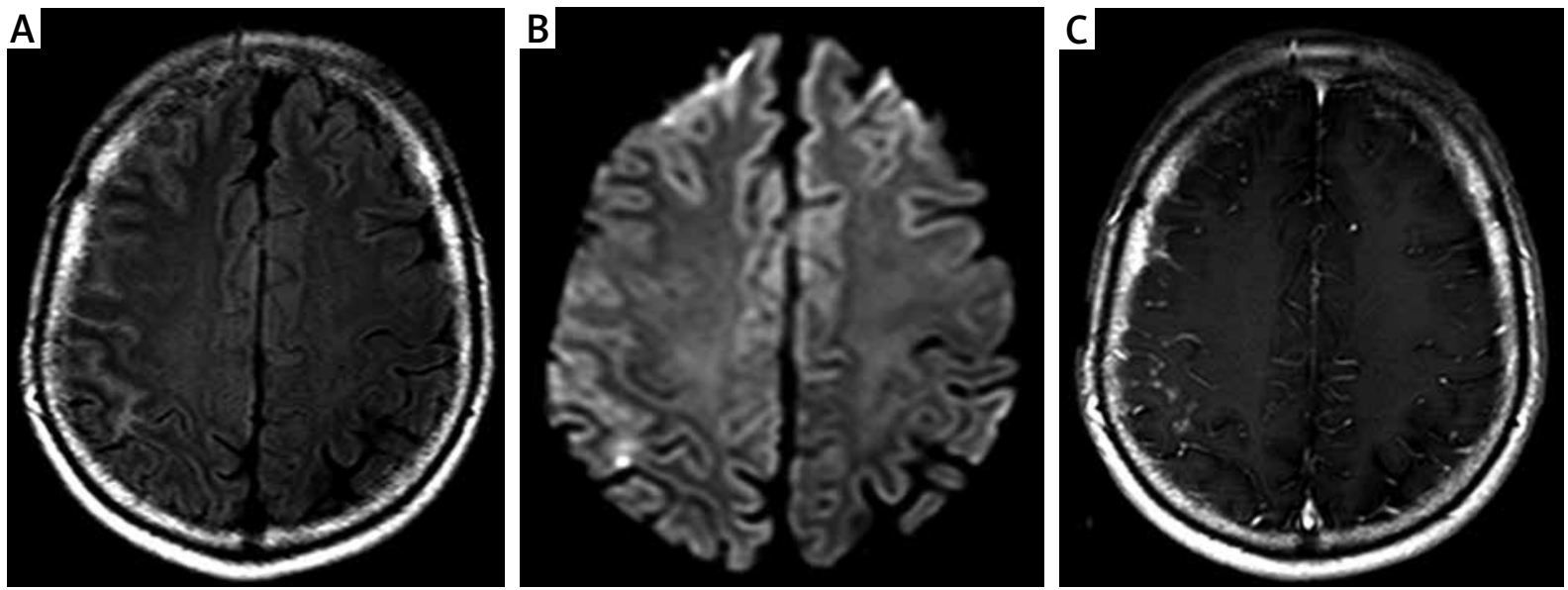

Fig. 1. MRI scan of the brain on first admission to the hospital. The study description was prepared by prof. Edyta Szurowska. Axial TIRM dark fluid sequence - cerebral cortex oedema in the frontoparietal region of the right hemisphere with clamping grooves of brain and increase of their signal (A); axial DWI sequence - minor foci of diffusion reduction in the right parietal lobe (B); axial, contrast-enhanced T1 dependent images- distinct enhancement of dura mater and pia mater in frontoparietal area (C).

neurology department with the suspicion of stroke. The patient suffered from coronary heart disease for seventeen years and RA for 10 years which was being treated with methylprednisolone ( $8 \mathrm{mg} /$ day) and methotrexate (20 mg/week). Neurological symptoms were preceded by severe headache, right tinnitus and increased blood pressure ( $\max 220 / 100 \mathrm{~mm} \mathrm{Hg}$ ). MRI of the head (Fig. 1) showed hyper-intense signaling and diffusion restriction from the fronto-parieto-temporal region, addition- ally contrast studies showed leptomeningeal enhancement in the right hemisphere.

Transcranial USG revealed atherosclerotic lesions that were hemodynamically insignificant which lead to exclusion of stroke. Lumbar puncture was performed for further assessment which revealed pleocytosis (7 cells/ $\mu \mathrm{l}$ ), increased level of protein and was positive for Candida mannan which lead to a suspicion of fungal meningitis. Moreover, presence of IgG oligoclonal bands were present

Table I. Laboratory results

\begin{tabular}{|lcc|}
\hline \multicolumn{3}{|c|}{ CSF analysis } \\
\hline Parameters tested & Result & $\begin{array}{c}\text { Reference } \\
\text { values }\end{array}$ \\
\hline Chlorides $(\mathrm{mmol} / \mathrm{l})$ & 115 & $115-130$ \\
\hline Albumin $(\mathrm{mg} / \mathrm{l})$ & 771.04 & $<350$ \\
\hline Total Protein $(\mathrm{g} / \mathrm{l})$ & 1.11 & $0.15-0.40$ \\
\hline Glucose $(\mathrm{mg} / \mathrm{dl})$ & 63 & $40-70$ \\
\hline Cell count $(\mathrm{cell} / \mathrm{s} / \mathrm{ll})$ & 8 & $<3$ \\
\hline Erythrocytes $($ per high power field) & $1-3$ & Absent \\
\hline Leukocytes (per high power field) & $0-1$ & Absent \\
\hline Borrelia antibodies & Negative & Negative \\
\hline Aspergillosis antibodies & Negative & Negative \\
\hline Candida mannan antibodies & Positive & Negative \\
\hline Immunoglobulin $(\mathrm{lgG})(\mathrm{mg} / \mathrm{l})$ & 36.10 & Absent \\
\hline
\end{tabular}

\begin{tabular}{|c|c|c|}
\hline \multicolumn{3}{|c|}{ Serology } \\
\hline Parameters tested & Result & $\begin{array}{c}\text { Reference } \\
\text { values }\end{array}$ \\
\hline Hemoglobin (g/dl) & 11.6 & $13-17$ \\
\hline Hematocrit (\%) & 35.8 & $40-50$ \\
\hline Basophils (\%) & 0.0 & $0-2$ \\
\hline Eosinophils (\%) & 0.0 & $0-6$ \\
\hline Neutrophils (\%) & 83.6 & $40-70$ \\
\hline Monocytes (\%) & 6.2 & $4-8$ \\
\hline Lymphocytes (\%) & 10.0 & $20-50$ \\
\hline MCV (fl) & 86.1 & $80-100$ \\
\hline Glucose (mg/dl) & 140 & $60-99$ \\
\hline CRP $(\mathrm{mg} / \mathrm{l})$ & 3.6 & $0-5$ \\
\hline ESR $(\mathrm{mm} / \mathrm{h})$ & 8 & $<15$ \\
\hline ACPA (U/ml) & 76 & $<5$ \\
\hline RF (IU/ml) & 229 & $<30$ \\
\hline
\end{tabular}

ACPA - anti-cyclic citrullinated peptide, CRP - C-reactive protein, CSF - cerebrospinal fluid analysis, ESR - erythrocyte sedimentation rate, $M C V$ - mean corpuscular volume, $R F-$ rheumatoid factor. 

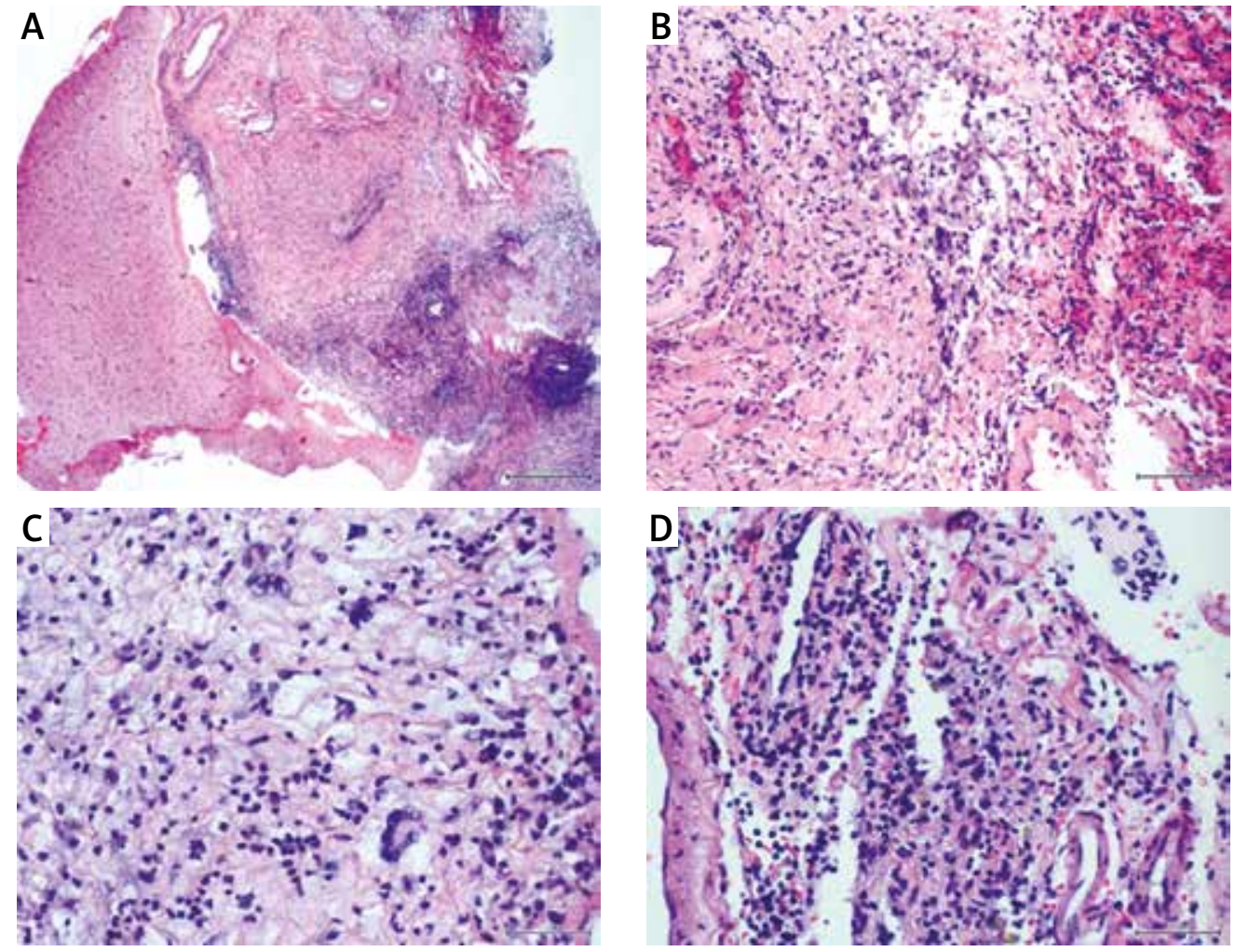

Fig. 2. Histopathology of cerebro-meningeal biopsy. The study description was prepared by prof. Ewa Iżycka-Świeszewska. Intense chronic inflammation with vasculitis and granulation tissue within leptomeninges and subarachnoid space as well as slight vasculitis of cortical vessels (HE, $100 \times)(A)$; fibrosing granulation tissue with histiocytic multinucleated cell necrotizing granuloma and hyalinization of blood vessels walls (HE, $200 \times)(B)$; inflammatory infiltrate made of foamy and multinucleated histiocytes, lymphocytes and plasmocytes (HE, $400 \times)(C)$; chronic meningitis with active vasculitis in form of dense intraparietal and perivascular lympho-plasmocytic infiltrates, old extravasations with hemosiderin- laden macrophages and collagen fibers deposition (HE, $400 \times)$ (D).

in both cerebrospinal fluid (CSF) and serum, suggesting passive diffusion between them. Additionally, a brain biopsy affirmed lymphoid infiltration of the meninges with histiocytes containing PAS+ substance. Due to suspicion of fungal etiology the patient was treated with fluconazole for 3 months, but clinical condition did not improve.

The patient reported back after 3 months with severe headache (VAS 10/10), dizziness, sleeplessness and mood disorders. Laboratory tests showed mild normocytic anemia (Hgb $11.6 \mathrm{~g} / \mathrm{dl}$ ), thrombocytopenia (PLT $122 \mathrm{G} / \mathrm{l}$ ), lymphopenia $(0,42 \mathrm{G} / \mathrm{l})$ and low inflammation markers (ESR $8 \mathrm{~mm} / \mathrm{h}$, CRP $3.6 \mathrm{mg} / \mathrm{l}$ ). Immunological assessment reveal the presence of anti-nuclear autoantibodies (ANA HEp-2 1 : 320 homogenous type, > 1 : 2560 speckled type; ANA-immunoblot: anti-SSA-Ro60 and anti-SSA-Ro52; anti-SS-A (154 IU/ml; $n<20.0 \mathrm{IU} / \mathrm{ml})$, RF $(229 \mathrm{IU} / \mathrm{ml}$, $n<30 \mathrm{lU} / \mathrm{ml})$ and ACPA antibodies (76 U/ml; $n<5 \mathrm{U} / \mathrm{ml})$. Anti-neutrophil antibodies (ANCA), anti-endothelial cell antibodies or antiphospholipid antibodies were negative (Table I). There were no symptoms of active vasculitis in other organs (physical examination, chest CT, abdominal USG, urine test). Follow-up MRI revealed no visible regression of previously described changes. Fungal etiology seemed unlikely in this situation.

The neuropathological consultation of brain samples (Fig. 2), combined with the clinical presentation and laboratory results allowed to diagnose chronic rheumatoid meningitis and CNS vasculitis in the course of RA. Additionally, the diagnosis of secondary Sjögren's syndrome (sSS) was based on a positive Schirmer's test, symptoms of dry mouth (unstimulated salivary flow) and presence of anti-SSA antibodies in the immunological assessment.

Immunosuppressive treatment with methylprednisolone (total $3 \mathrm{~g} \mathrm{IV}$ ), followed by oral prednisone $0.5 \mathrm{mg} / \mathrm{kg}$ QD and IV cyclophosphamide every 3 weeks for 8 months (total dose $7.2 \mathrm{~g}$ ) was administered. Currently after 4 years the patient is in good condition with 

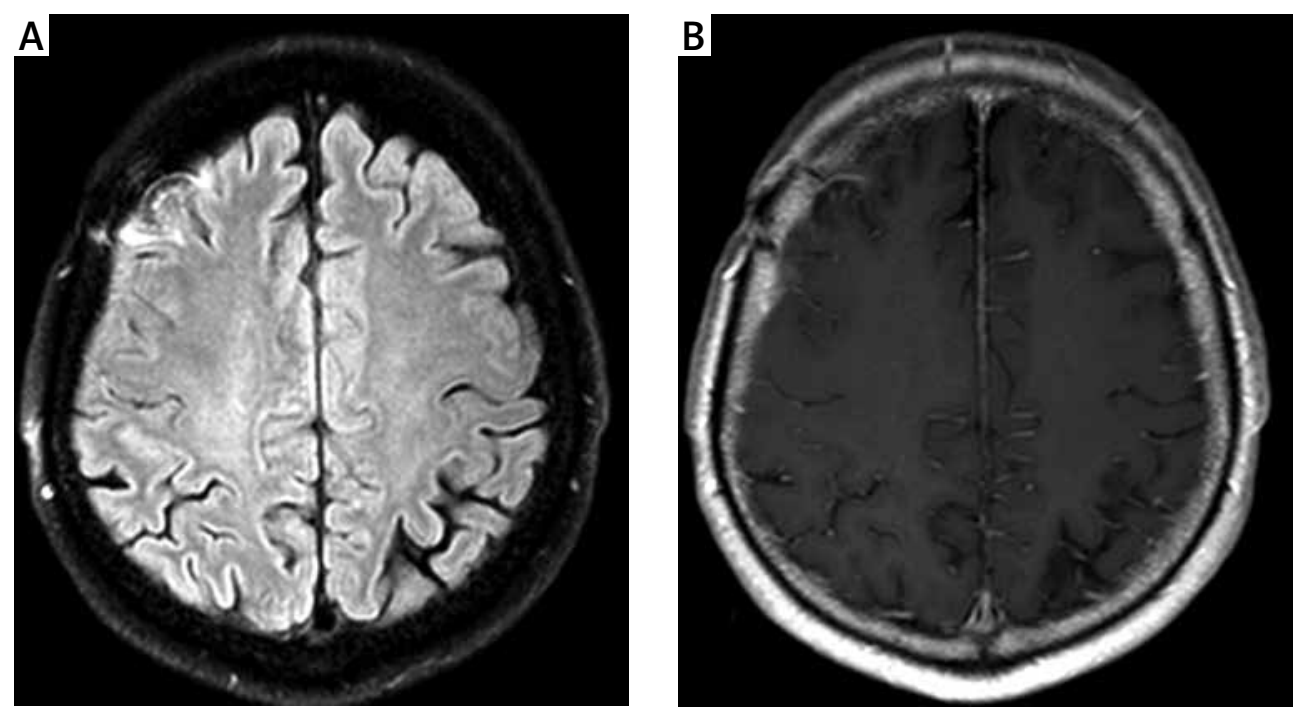

Fig. 3. MRI scan of the brain after 6 months of immunosuppressive treatment. The study description was prepared by prof. Edyta Szurowska. Axial FLAIR sequence - regression of cerebral cortex oedema placed in the frontoparietal region of the right hemisphere, grooves of brain of proper width, visible only band-like area of gliosis in the area of surgical access (A); axial, contrast-enhanced T1 - dependent images - distinct regression of meninges enhancement in frontoparietal area, minor enhancement of post-biopsy site (B).

episodic headaches of low intensity (VAS 2/10). The control MRI (Fig. 3) revealed a decrease in temporal enhancement of the meninges. The patients were treated with methotrexate in current dose $15 \mathrm{mg}$ per week.

\section{Results of data searching}

We analyzed similar clinical problems in the literature. Since 2012 there have been 28 different case reports published about pachymeningitis in a course of RA. Out of these, 5 of them reported patients with seronegative RA in whom pachymeningitis was the first clinical manifestation of the autoimmune disease (Fig. 4) [3-8].

\section{Discussion}

Pachymeningitis is the inflammation of the most superficial layer of the meninges-dura mater [1]. In some cases, the inflammation can reach the leptomeninges and the brain parenchyma [3]. The etiology of pachymeningitis ranges from infectious causes (like syphilis, fungal or viral), lymphomas, drug-induced to auto-immune (like vasculitis or RA) and IgG4-related pathologies $[4,9]$. Extremely rare cause of hypertrophic pachymeningitis is primary Sjögren's syndrome (SS). Nakano et al. [10] report only a single case of patient with primary SS who developed pachymeningitis. There have not been any cases of patients with secondary SS associated with pachymeningitis. This variety of causes lead to considerable diagnostic difficulties. Identifying the cause of changes in the CNS is crucial to implement proper treatment and avoid complications.

Cervical myelopathy due to atlanto-axial subluxation is the most well-known CNS manifestation in the course of RA, others include spinal cord compression, subdural hematoma [11], cerebral vasculitis, rheumatoid nodule formation and pachymeningitis [12]. The first suggestion of pachymeningitis due to RA was by Wells [13] in 1969, since then there have been a number of reviews and reports which showed CNS involvement in RA patients.

While common neurological symptoms like headaches, seizures, focal neurological deficits, altered mental condition and cranial nerve palsies that present as blindness or sensorineural deafness were reported over the years $[12,14]$, some rare symptoms have been described in the recent times which include neuropsychiatric presentation [5] and acute parkinsonism [15]. Due to these varied neurological symptoms and a multitude of etiologies for pachymeningitis, diagnosis of rheumatoid meningitis is challenging.

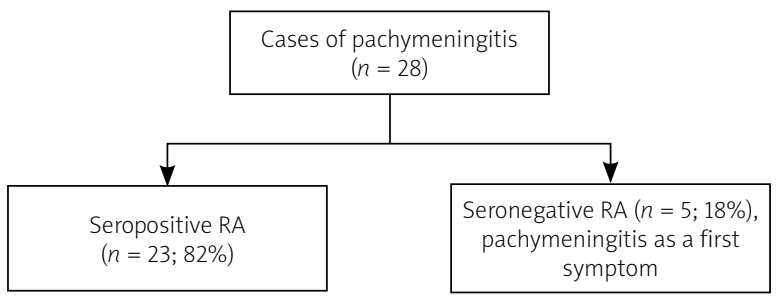

Fig. 4. Results of data searching. 
Diagnosis of RM depends on a combination of clinical presentation, lumbar puncture, brain MRI and biopsy along with exclusion of other etiologies [3]. The CSF analysis on lumbar puncture may have mild pleocytosis with predominant lymphocytes, increased protein and increased or normal glucose. To exclude any infections, CSF cultures should be negative.

In our case, the cultures were negative for bacteria, borreliosis and aspergillosis, but was positive for Candida mannan due to which a suspicion of fungal etiology arose. MRI with DWI sequence shows meningeal thickening and sometimes there maybe presence of rheumatoid nodules with contrast enhancement. But these tests are non-specific for RM and a biopsy for establish the diagnosis is necessary. In the histopathological assessment were nonspecific meningeal inflammation, presence of nodules and vasculitis [16].

Magaki et al. [6] stated that the most characteristic histological findings in brain biopsy of described patients are picture of pachymeningitis or leptomeningitis with rheumatoid nodules (typical presentation) and vasculitis affected a smaller parenchymal and meningeal arteries. The mononuclear cells, especially plasma cells, multinucleated giant cells, neutrophils (less commonly) and occasional necrosis formed inflammatory infiltrates. The authors suggested that the autoimmunity of collagen may be a main cause of inflammatory changes in the meninges.

Serological findings in a patient with RM include elevated CRP, ESR, RF and ACPA antibodies, out of these the latter two are considered specific, all of which were elevated in our patient [1]. ACPA antibodies titer has been a novel tool to check the disease advancement as its presence is associated with severe extra-articular manifestations and progressive damage to the joint [17] Moreover, some case reports suggest presence of RF in the CSF as a possible diagnostic finding, although we did not assess this in our patient [17]. Currently there are no specific biomarkers or guidelines to detecting RM.

Tay et al. [18] conducted a meta-analysis assessing association of anti-N-methyl-D-aspartate (anti-NMDA) receptor antibodies and neuropsychiatric (NP) syndromes in systemic lupus erythematosus (SLE) and SS which added another potential serological marker [19]. Their study showed that presence of anti-NMDA receptor antibodies in the serum could be used in diagnosing NP syndromes in SLE and SS, but it did not help in distinguishing the type of NP manifestation. However, no such data is available for patients with RA or other rheumatological diseases which also has NP manifestations and further studies need to be done to establish anti-NMDA antibody as a promising diagnostic marker.

No definitive treatment for RM currently exists due to its rarity and diverse clinical presentation but previ- ous case reports and studies have used a combination of corticosteroids, immune modulating drugs, cyclophosphamide and rituximab successfully [19-21]. However occurrence of PM in the course of treatment with biologics was also described [22].

Most cases of RM have occurred in patients with seropositive RA, however this may occur in patients at any stage of the disease $[6,8]$. In some case reports, RM was observed in patients as the first clinical manifestation of RA $[4,23]$. In our case, the patient had been diagnosed with seropositive RA for 10 years.

The pathogenesis of remains unclear, although many case reports over the years suggest the use of tumor necrosis factor inhibitor as a treatment for RA was the cause of RM [24-27]. It is hypothesized that these drugs induce development of accelerated nodulosis by various mechanisms like induction of cytokine expression modulation in particularly interferon [25].

Other theories include failure of inhibition of apoptosis in component cells or failure of interferon gamma inhibition which could promote granulomatosis [23]. Our patient was being treated with glucorticosteroids and methotrexate which may be the reason for development of RM as suggested by Huys et al. [25] in their study.

We analyzed 28 different case reports published about pachymeningitis due to RA. Five of them described patients without diagnosis of RA at the beginning with symptoms of pachymeningitis as the first clinical manifestation of the autoimmune disease [3-8] although both the patients in the Magaki et al. [6] report had a strong family history of RA. Most recurring clinical presentation in all these cases are stroke like symptoms, headaches, speech impairment, recurring seizures and most of these manifestations are progressive over a period of weeks to months. Thus, such varied presentation over a period leads to misdiagnosis.

The rest of the articles report cases in patients with seropositive RA most of whom were being treated for it with a combination of methotrexate and corticosteroids additionally some of them were being treated with TNF- $\alpha$ blockers [20, 22, 24-27] sulfasalazine [28, 29], a gold salt [30], iguratimod, a methanesulfonanilide [14, 31].

The imaging results rarely revealed pathology in CT scans, but findings in MRI aided towards the diagnosis of RM. Most of the cases showed abnormal signaling in MRI without contrast, it was necessary to perform contrast MRI to visualize the lepto- or pachymeningeal uptake and enhancement.

Other MRI sequences (FLAIR, DWI) showed hyperintense signals over the meninges. Some cases the first MRI performed did not show any pathological abnormalities [24, 32, 33] but after progression of the disease similar findings were noted. Unfortunately, there are no findings that exist in imaging which are specific for RM, 
so imaging is useful in excluding etiologies like malignancies, ischemic stroke or hemorrhage.

Laboratory tests, which include serum inflammatory markers (CRP, ESR), were persistently elevated in most of the patients leaving 13 cases where these were either not reported or were normal. But these inflammatory markers can be elevated in several systemic diseases. CSF findings were persistent in almost all the cases with pleocytosis and increased protein content as the characteristic findings leaving 2 cases where it was normal $[5,30]$.

In some cases there was presence of RF or ACPA $[3,34,35]$ antibodies in undiluted CSF therefore presence of RF or ACPA antibodies in the CSF could be a good diagnostic marker of RM, but these are not present in all the cases. Current biomarkers include serum RF, ACPA and these findings are quite persistent in RM patients with either one or both being present in all the 29 cases.

In addition to these, Tsuzaki et al. [24] reported elevated antinuclear antibodies (ANA), Anti-Sjögren's-syndrome-related antigen A/Anti-Ro (SS-A), Anti-La (SS-B) antibodies, chemokine ligand 13 (CXCL13) and Akamatsu et al. [36] reported elevated interleukin 6 (IL-6) while one patient had negative serum RF, ANA and ANCA [4]. Elevated IL- 6 has been reported very rarely in previous literature. Biopsy findings in all the cases are very similar and lead to confirmed diagnosis of RM which typically showed granulomatous inflammation of the meninges with lymphoplasmacytic infiltrate, perivascular inflammation and presence of rheumatoid nodules in some cases. As of today, meningeal biopsy is the most accurate diagnostic test for RM, but due to its' invasive nature it shouldn't be performed unless all other etiologies have been excluded.

Preferred treatment method was intravenous methylprednisolone followed by oral prednisolone. Treatment for RA was continued or was changed to rituximab which successfully managed the RA progression [1, 3, 7, 25, 35]. Elevated serum ACE and $\beta-2$ microglobulin were reported by Pellerin et al. [15] and McKenna et al. [8] although, these findings haven't been clearly associated with RM $[8,15]$. Some patients had pulmonary manifestations in the form of nodules or pleural effusions which were attributed as extra-articular manifestations of RA after examinations [22, 32, 35].

\section{Conclusions}

Previously RM was identified during autopsies, but recently due to the advancement in imaging techniques and diagnostic procedures, it has been possible to detect it early and start the appropriate treatment. This review establishes that the incidence of pachymeningitis in the course of RA is quite rare but is still increasing and there is a need to find more specific tests or biomarkers which could facilitate differential diagnostics of this disease.

\section{Acknowledgements}

The authors want to thank prof. Ewa lżycka-Świeszewska and prof. Edyta Szurowska for their help in preparing histopathology and imaging tests.

The authors declare no conflict of interest.

\section{References}

1. Scheitel M, Ives ST, Nasr R, Nolan MW. When the Plot Thickens: A Rare Complication of Rheumatoid Arthritis. J Community Hosp Intern Med Perspect 2019; 9: 143-146, DOI: 10.1080/20009666.2019.1593780.

2. Muravyev YV. Extra-articular Manifestations of Rheumatoid Arthritis. Nauchno-Prakticheskaya Revmatol 2018; 56: 356362, DOI: 10.14412/1995-4484-2018-356-362.

3. Lee Ching C, Kenyon L, Berk M, Park C. Rheumatoid Meningitis Sine Arthritis. J Neuroimmunol 2019; 328: 73-75, DOI: 10.1016/j.jneuroim.2018.12.001.

4. Parsons AM, Zuniga LA, Hoxworth JM, et al. Rheumatoid Meningitis: A Case Review. Neurologist 2018; 23: 83-85, DOI: 10.1097/NRL.0000000000000158.

5. Lubomski M, Sy J, Buckland M, et al. Rheumatoid Leptomeningitis Presenting With an Acute Neuropsychiatric Disorder. Pract Neurol 2019; 19: 68-71, DOI: 10.1136/practneurol-2018-001978.

6. Magaki S, Chang E, Hammond RR, et al. Two Cases of Rheumatoid Meningitis. Neuropathology 2016; 36: 93-102, DOI: 10.1111/neup.12238.

7. Schuster S, Braass H, Iking-Konert C, et al. Rheumatoid Meningitis: A Rare Cause of Aseptic Meningitis With Frequently Stroke-Like Episodes. Neurol Clin Pract 2018; 8: 451-455, DOI: 10.1212/CPJ.0000000000000504.

8. McKenna MC, Vaughan D, Bermingham N, Cronin S. Rheumatoid Arthritis Presenting as Rheumatoid Meningitis. BMJ Case Rep 2019; 12: bcr-2018-226649, DOI: 10.1136/bcr-2018226649.

9. Wallace ZS, Carruthers MN, Khosroshahi A, et al. IgG4-related Disease and Hypertrophic Pachymeningitis. Medicine (Baltimore) 2013; 92: 206-216, DOI: 10.1097/MD.0b013e31829cce35.

10. Nakano Y, Yamamoto M, Komatsu K, et al. Hypertrophic Pachymeningitis in Sjögren's Syndrome. Intern Med 2018; 57: 413-415, DOI: 10.2169/internalmedicine.9406-17.

11. Yagita K, Shinde A, Suenaga T. Rheumatoid meningitis can present MRI findings that mimic chronic subdural haematoma. BMJ Case Rep 2019; 12: e229642, DOI: 10.1136/bcr2019-229642.

12. Tan HJ, Raymond AA, Phadke PP, Rozman Z. Rheumatoid pachymeningitis. Singapore Med J 2004; 45: 337-339. 
13. Wells AL. Extra-articular manifestations of rheumatoid arthritis. Br Med J 1969, 4: 173, DOI: 10.1136/bmj.4.5676.173-c.

14. DeQuattro K, Imboden JB. Neurologic Manifestations of Rheumatoid Arthritis. Rheum Dis Clin North Am 2017; 43: 561-571, DOI: 10.1016/j.rdc.2017.06.005.

15. Pellerin D, Wodkowski M, Guiot MC, et al. Rheumatoid Meningitis Presenting With Acute Parkinsonism and Protracted Non-convulsive Seizures: An Unusual Case Presentation and Review of Treatment Strategies. Front Neurol 2019; 10: 163, DOI: 10.3389/fneur.2019.00163.

16. Grose D, Linger M, Tinni S, Sahathevan R. Rheumatoid meningitis: a rare cause of unilateral pachymeningitis. BMJ Case Rep 2019; 12, e227905, DOI: 10.1136/bcr-2018-227905.

17. Kim HY, Park JH, Oh HE, et al. A case of rheumatoid meningitis: Pathologic and magnetic resonance imaging findings. Neurol Sci 2011; 32: 1191-1194, DOI: 10.1007/s10072-011-0727-9.

18. Tay SH, Fairhurst AM, Mak A. Clinical utility of circulating anti$\mathrm{N}$-methyl- $\mathrm{d}^{-a s p a r t a t e}$ receptor subunits NR2A/B antibody for the diagnosis of neuropsychiatric syndromes in systemic lupus erythematosus and Sjögren's syndrome: An updated meta-analysis. Autoimmun Rev 2017; 16: 114-122, DOI: 10.1016/j.autrev.2016.12.002

19. Degboé Y, Fajadet B, Laurent C, et al. A rare case of rheumatoid pachyleptomeningitis successfully treated with rituximab. Rheumatology (Oxford) 2017; 56: 1238-1240, DOI: 10.1093/ rheumatology/kex059.

20. Harrison NS, Kishore S, Majithia V. Rheumatoid meningitis: successful remission with rituximab. BMJ Case Rep 2018; 11: e226642, DOI: 10.1136/bcr-2018-226642.

21. Moeyersoons A, Verschueren P, Tousseyn T, De Langhe E. Rheumatoid granulomatous disease and pachymeningitis successfully treated with rituximab. Acta Clin Belg 2018; 73: 307-312, DOI: 10.1080/17843286.2017.1375193.

22. Gherghel N, Stan A, Stan H. Pearls \& Oy-sters: Rheumatoid meningitis occurring during treatment with etanercept. Neurology 2018; 91: 806-808, DOI: 10.1212/WNL.0000000000006397.

23. Atzeni F, Talotta R, Masala IF, et al. Central nervous system involvement in rheumatoid arthritis patients and the potential implications of using biological agents. Best Pract Res Clin Rheumatol 2018; 432: 500-510, DOI: 10.1016/j. berh.2019.02.003

24. Tsuzaki K, Nakamura T, Okumura H, et al. Rheumatoid Meningitis Occurring during Etanercept Treatment. Case Rep Neurol Med 2017; 2017: 7638539, DOI: 10.1155/2017/7638539.
25. Huys AC, Guerne PA, Horvath J. Rheumatoid meningitis occurring during adalimumab and methotrexate treatment. Joint Bone Spine 2012; 79: 90-92, DOI: 10.1016/j.jbspin.2011.07.008.

26. Koopmansch C, D'Haene N, Hastir D, et al. A 63-Year-Old Woman with a Right Frontal Lesion. Brain Pathol 2016; 26 : 555-556, DOI: 10.1111/bpa.12393.

27. Nihat A, Chinthapalli K, Bridges L, et al. Rheumatoid meningitis. Pract Neurol 2016; 16: 312-324, DOI: 10.1136/practneurol-2015-001306.

28. Roy B, Uphoff DF, Silverman IE. Rheumatoid Meningitis Presenting With Multiple Strokelike Episodes. JAMA Neurol 2015; 72: 1073-1076, DOI:10.1001/jamaneurol.2015.1105.

29. Mason A, Chan C, Pengas G, Holroyd C. Rheumatoid pachymeningitis: a rare extra- articular manifestation of rheumatoid arthritis. Rheumatol Adv Pract 2018; 2 (Suppl 1), rky033.023, DOI: 10.1093/rap/rky033.023.

30. Lu L, Chwalisz B, Pfannl R, Narayanaswami P. Rheumatoid meningitis: a rare complication of rheumatoid arthritis. BMJ Case Rep 2015; 2015: bcr2014208745, DOI: 10.1136/bcr2014-208745

31. Matsuda S, Yoshida S, Takeuchi T, et al. Asymptomatic rheumatoid meningitis revealed by magnetic resonance imaging, followed by systemic rheumatic vasculitis: A case report and a review of the literature. Mod Rheumatol 2019; 29: 370-376, DOI: 10.1080/14397595.2016.1232333.

32. Duray MC, Marchand E, Gohy S, et al. Granulomatous meningitis due to rheumatoid arthritis. Acta Neurol Belg 2012; 112: 193-197, DOI: 10.1007/s13760-012-0021-5.

33. Krys। D, Zamecnik J, Senolt L, Marusic P. Chronic repetitive nonprogressive epilepsia partialis continua due to rheumatoid meningitis. Seizure 2013; 22: 80-82, DOI: 10.1016/j.seizure.2012.10.006

34. Choi SJ, Ho Park Y, Kim JA, et al. Pearls \& Oy-sters: Asymmetric meningeal involvement is a common feature of rheumatoid meningitis. Neurology 2017; 88: e108-e110, DOI: 10.1212/ WNL.0000000000003744.

35. Nissen MS, Nilsson AC, Forsberg J, et al. Use of Cerebrospinal Fluid Biomarkers in Diagnosis and Monitoring of Rheumatoid Meningitis. Front Neurol 2019; 10: 666, DOI: 10.3389/ fneur.2019.00666.

36. Akamatsu M, Maki F, Akiyama $\mathrm{H}$, et al. Rheumatoid meningitis presenting with a stroke-like attack treated with recombinant tissue plasminogen activator: a case presentation. BMC Neurol 2018; 18: 139, DOI: 10.1186/s12883-018-1143-z. 